\title{
The Segmented Aperture Interferometric Nulling Testbed (SAINT) I: overview and air-side system description
}

\author{
Brian A. Hicks ${ }^{*} a, b$, Richard G. Lyon ${ }^{b}$, Peter Petrone $\mathrm{III}^{c}$, \\ Marlin Ballard ${ }^{a}$, Matthew R. Bolcar ${ }^{b}$, Jeff Bolognese ${ }^{b}$, Mark Clampin ${ }^{b}$, \\ Peter Dogoda ${ }^{c}$, Daniel Dworzanski ${ }^{d}$ Michael A. Helmbrecht ${ }^{e}$, Corina Koca ${ }^{b}$, Ron Shiri ${ }^{b}$ \\ ${ }^{a}$ University of Maryland, College Park, MD, USA \\ ${ }^{b}$ NASA Goddard Space Flight Center, Greenbelt, MD, USA \\ ${ }^{c}$ Sigma Space Corporation, Lanham, MD, USA \\ ${ }^{d}$ Optimax Systems, Inc., Rochester, NY, USA \\ ${ }^{e}$ Iris AO, Inc., Berkeley, CA, USA
}

\begin{abstract}
This work presents an overview of the Segmented Aperture Interferometric Nulling Testbed (SAINT), a project that will pair an actively-controlled macro-scale segmented mirror with the Visible Nulling Coronagraph (VNC). SAINT will incorporate the VNC's demonstrated wavefront sensing and control system to refine and quantify end-to-end high-contrast starlight suppression performance. This pathfinder testbed will be used as a tool to study and refine approaches to mitigating instabilities and complex diffraction expected from future large segmented aperture telescopes.
\end{abstract}

Keywords: Exoplanets, high-contrast imaging, nulling interferometry, wavefront sensing and control, space telescopes, segmented mirrors

\section{INTRODUCTION}

Direct detection and characterization of exoplanets is predicated on collecting area and angular resolution available with large telescopes, as well as the ability to suppress starlight relative to the planet at small angular separations using coronagraphic techniques. Recent yield studies indicate that an aperture of 8 meters or greater is necessary to discover tens of Earth-sized habitable zone (HZ) exoplanets, thus allowing for a statistical analysis of the likelihood of habitability. ${ }^{1}$ Contrast, resolution, and photon count rates for hypothetical Earth-like exoplanets orbiting nearby stars observed at a central wavelength of $\lambda=760 \mathrm{~nm}$ with a $12-\mathrm{m}$ telescope are presented in Fig. 1. The center and right top panels of Fig. 1 show the detection space for observing the mean HZ around these stars assuming a yet to be demonstrated high-contrast field of view spanning $3 \lambda / D$ inner working angle (IWA) to $30 \lambda / D$ outer working angle (OWA). The lower left panel of Fig. 1 shows visible light planet-to-star luminosity ratios of hypothetical Earth-like exoplanets orbiting stars within 30 parsecs plotted versus residual diffracted starlight in the image planet at the mean orbital separation of each star's HZ assuming $\Theta^{4}$ suppression of calculated stellar angular diameters shown in the upper right panel. The lower center and right plots show the expected photon count rates $\left(\phi_{p}\right)$ of the planets and expected signal to noise for a single exposure with noiseless detector assuming an integration time equal to $1 / \phi_{p}$, respectively.

Complex diffraction is problematic for many coronagraphic designs and most high contrast results to date have used unobscured circular apertures, nominally demonstrating capability for future pairing with monolithic offaxis telescopes. In 2018, the James Webb Space Telescope (JWST) will have the largest space telescope aperture at 6.5 meters. JWST is a deployable segmented aperture telescope, and NASAs investment in JWST will have an impact on telescope architectures for future flight missions; apertures of $\geq 8-\mathrm{m}$ are most likely to follow the segmented aperture approach. Thus, coronagraphy should be adept at operating on segmented apertures, and several approaches have accordingly been undergoing development for exoplanet detection using both groundand space-based segmented telescopes. These approaches include aperture masking, ${ }^{2}$ beam shaping, ${ }^{3}$ and nulling

\footnotetext{
*bahicksmail@gmail.com
} 

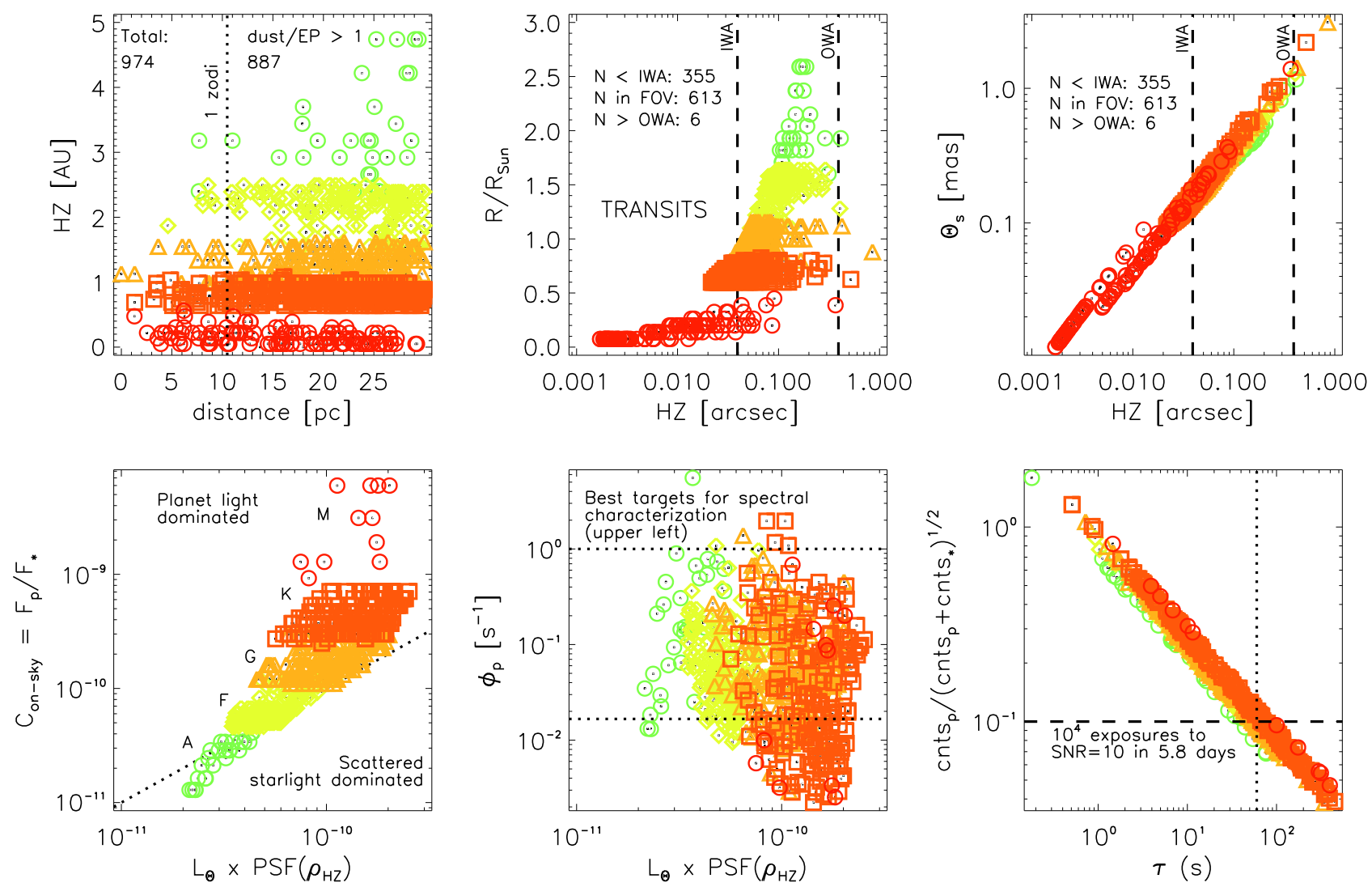

Figure 1. Accessibility of habitable zones around A, F, G, K, and M stars within 30 pc of Earth based on system resolution, contrast, and photometric capability with the following assumptions: $12-\mathrm{m} 3$-ring hexagonal aperture Central $\lambda=0.76 \mu \mathrm{m}$ $\left(\mathrm{O}_{2}\right) 10 \%$ spectral bandwidth $5 \%$ total throughput $\theta^{4}$ stellar suppression $\mathrm{D} / 7$ linear shears 0.5 mas pointing $3 \lambda / D$ inner working angle (IWA) $30 \lambda / D$ outer working angle (OWA) $\mathrm{PSF} \propto \rho^{-2.5}$ Approximately $10-20$ of the systems plotted will transit. Further description of the plots shown in this figure may be found in the text.

interferometry. ${ }^{4}$ Nulling stands out as an option that has been shown to be adaptable to a variety of apertures considered during the 2008 Astrophysics Strategic Mission Concept (ASMC) studies. Three of the ASMC studies included a VNC: the Extrasolar Planetary Imaging Coronagraph (EPIC) with a filled aperture, ${ }^{5}$ the Advanced Technology Large Aperture Space Telescope (ATLAST) with a segmented aperture, ${ }^{6}$ and the Dilute Aperture Visible Nulling Coronagraphic Imager (DAViNCI) with a sparse aperture. ${ }^{7}$

This work presents an overview of the Segmented Aperture Interferometric Nulling Testbed (SAINT), a project that will pair an actively-controlled macro-scale segmented mirror with the Visible Nulling Coronagraph (VNC). SAINT will use the VNC's demonstrated wavefront sensing and control system to refine and quantify the end-to-end system performance for high-contrast starlight suppression. This pathfinder system will be used as a tool to study and refine approaches to mitigating instabilities and complex diffraction expected from future large segmented aperture telescopes. SAINT introduces a fine pointing system (FPS) to the VNC, and both will operate inside a vacuum chamber to provide an isolated environment for demonstrations at atmospheric pressure, at reduced pressure similar to a stratospheric balloon environment, or at high vacuum to emulate system operation in a space-like environment. This overview of the SAINT system and the project goals is provided along with a more detailed description of the air-side optics and initial measurements taken following their fabrication and alignment. 


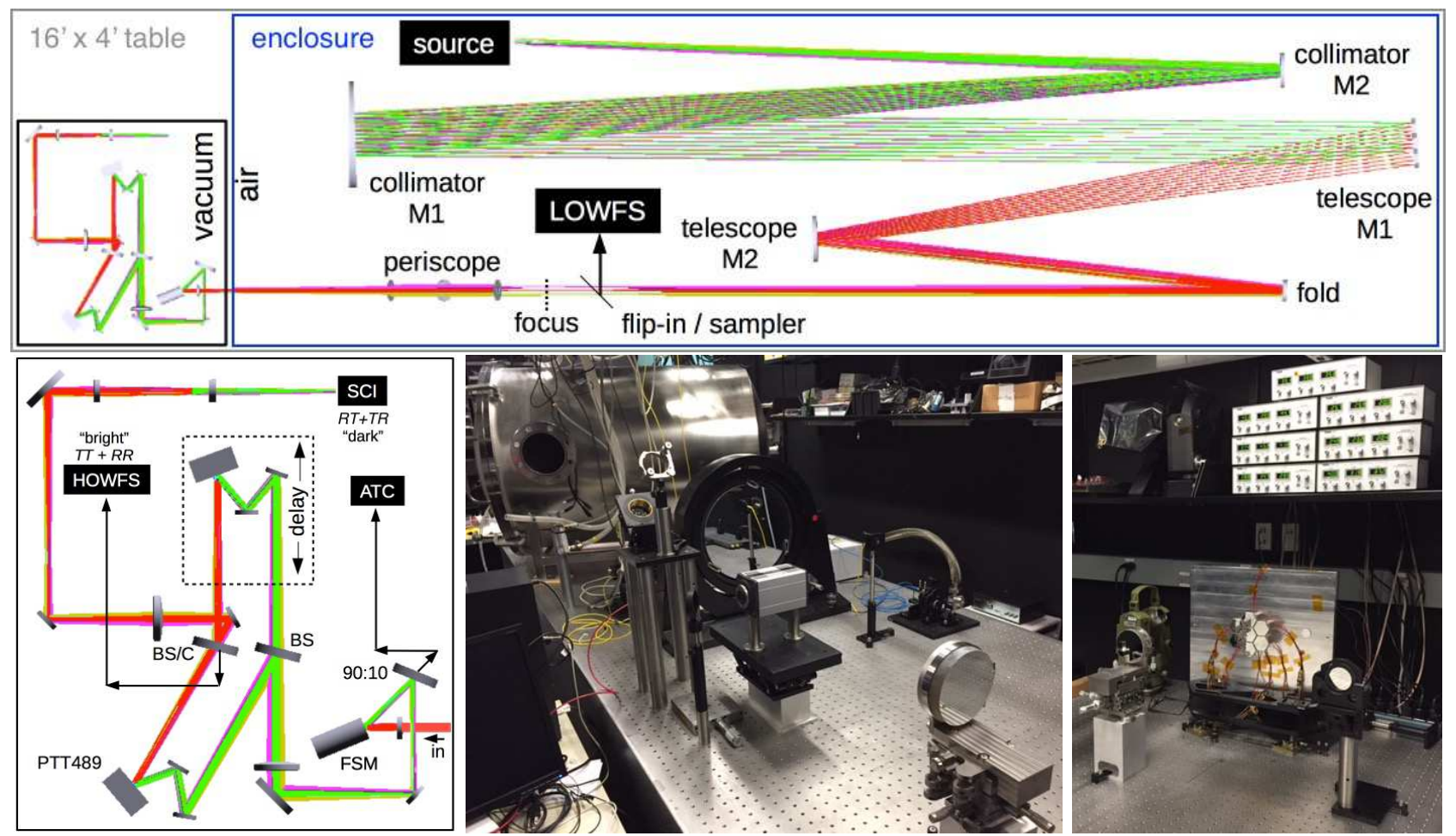

Figure 2. An end-to-end raytrace of the Segmented Aperture Interferometric Nulling Testbed (SAINT) consisting of 4 primary subsystems: (i) Source Module, (ii) Segmented Aperture Telescope, (iii) Visible Nulling Coronagraph, and (iv) Control System (not shown). SAINT will demonstrate high-contrast imaging with a segmented telescope, develop coupled active mirror sensing and control, and assess closed-loop contrast and IWA over a finite spectral bandpass of $\Delta \lambda=20 \mathrm{~nm}$ centered at or near $633 \mathrm{~nm}$. The optics are shown to scale, and the table, vacuum chamber, and enclosure are shown approximately to scale. A pupil is traversed each time the ray bundle changes color from predominantly red to green and vice versa.

\section{SAINT OBJECTIVES}

In support of NASAs Exoplanet Exploration Program and the Technology Demonstration for Exoplanet Missions (TDEM) component of NASAs Strategic Astrophysics Technology (SAT) program, the VNC is being coupled via a FPS with an actively controlled segmented primary mirror telescope to form SAINT, which is shown in Fig. 2. The SAINT project objective is to achieve and hold a contrast of $10^{8}$ at an IWA of $4 \lambda / D$ within a bandpass of $\Delta \lambda=20 \mathrm{~nm}$ centered at $633 \mathrm{~nm}$ for 1,000 seconds on three separate occasions, with a goal of $10^{9}$ at an IWA of $3 \lambda / D$ in a bandpass $\Delta \lambda=40 \mathrm{~nm}$. Satisfying this TDEM Milestone objective is the next step in establishing the VNC as a viable option for a future large space telescope requiring capability for directly detecting and characterizing Earth-like exoplanets. The project will entail making broadband contrast measurements, which will require broadband wavefront sensing and control. The project builds on architecture developed for the VNC TDEM Milestone \#1 demonstration of $10^{8}$ contrast at an IWA of $2 \lambda / D$ in narrowband light, ${ }^{8}$ and the Milestone \#2 effort to increase contrast and spectral bandpass through the inclusion of achromatic phase shifters (APS). ${ }^{9}$

The milestone represents a feasible objective based on current VNC Technology Readiness Levels (TRLs), and the TRL of component technologies, including wavefront sensing and control (WFS/C) with a segmented aperture and deformable mirror (DM), FPS, and APS. This project takes an important step towards enabling exoplanet coronagraphy for segmented apertures and ultimately for future missions such as a Large UV/Optical/Infrared (LUVOIR) telescope ${ }^{10}$ or the Habitable Exoplanet Imager (HabEx). ${ }^{11}$ Upon completion of this effort, sub-system technology will be properly positioned to address the additional steps required to mature this high-contrast imaging approach to TRL 6. An overview of the SAINT system is provided below along with a more detailed description of the air-side optics, their fabrication, alignment, and initial measurements characterizing system stability. 


\section{SYSTEM OVERVIEW}

Fig. 2 shows a layout of the SAINT optical system. A $16 \times 4$ stabilized air table supports the main components consisting of a source module and the segmented aperture telescope (see Fig. 4) housed inside an enclosure, and a vacuum chamber containing the VNC. The air-side segmented telescope component of SAINT is adapted from a sparse aperture phased array demonstrator, the Fizeau Interferometry Testbed (FIT). ${ }^{12,13}$ The telescope optics form one of two selectable branches of a relay, the other being a bypass that delivers a circular aperture with Gaussian intensity profile.

A vacuum window and baffle form the optical port that passes the light from the external segmented aperture telescope to the FSM for injection into the VNC. The VNC may be operated inside the chamber at atmospheric pressure, at reduced pressure similar to a stratospheric balloon environment, or at high vacuum to emulate system operation in a space-like environment. The vacuum chamber rests atop the optical table on passive isolators. Inside the chamber, the VNC breadboard rests on a shelf on additional passive isolators. A control system consisting of a computer, amplifier and driver electronics, and detector frame grabbers are being upgraded to be used in a real time operating system (RTOS) version of Linux running C with message passing interface (MPI) for threading across multiple processors. The control electronics are suspended from a rack above the optical table and vacuum chamber. Vacuum feedthroughs consisting of electrical cables and water chiller lines to cool the high-contrast focal plane detector pass through multiple tank bulkheads.

As shown in the Fig. 2 raytrace, an image of the segmented primary is relayed to a fast steering mirror (FSM). The FSM is controlled using feedback from an angle tracker camera (ATC) to form the FPS used to mitigate pointing drift and jitter between the telescope and VNC. Work on the FPS has begun and closed-loop performance will be presented in a future work. During the project, development of the telescope automatic phasing routines and upgrades to the VNC are to be performed parallel, and operation of the two subsystems will be demonstrated independently prior to operating the end-to-end system whereupon the FPS will be optimized.

The Lyot mask for the SAINT end-to-end system is shown in the left panel of Fig. 3, formed by the union of an Iris AO, Inc. PTT489 MMA (a device with 163 segments corresponding to seven hexagonal array rings less vertices) with the SAINT seven segment hexagonal primary mirror. A cross-section of the corresponding PSF (the right panel of Fig. 3 with intensity stretched as $I^{1 / 4}$ ) is shown in the central panel of the same figure. Inner sidelobes at $3 \lambda / D$ are attributed to the SAINT primary. The outer sidelobes are attributed to the seven-ringed MMA hexagonal array. Segments that span primary mirror gaps will be masked out and not controlled, leaving a total of 127 controllable segments. An e-beam deposition or photolithographic Lyot mask fabrication process will increase segment subaperture throughput by as much as $80 \%$ of the current throughput (i.e., $1.8 \times$ increase in area).
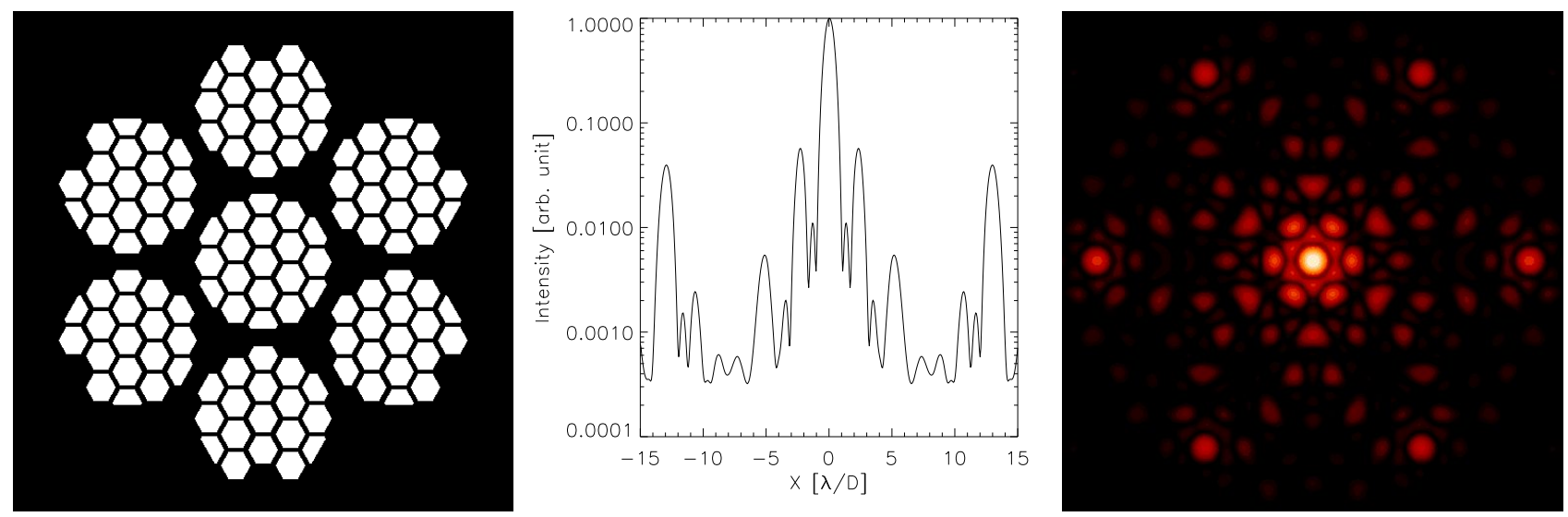

Figure 3. The SAINT Lyot mask (left) and corresponding cross-section (center) and $I^{1 / 4}$ stretch (right) of the PSF showing inner and outer sidelobes attributed to the SAINT primary and the seven-ringed MMA hexagonal array. 


\subsection{Segmented Aperture Telescope}

Components from an active sparse aperture testbed ${ }^{13}$ have been used to build the SAINT collimator and telescope (see photos in Fig. 2), including the new hexagonal array active primary. The primary (see Fig. 4) consists of seven hexagonal segments - a ring of six segments around a central segment that could be masked. Each segment is separately bonded to a flexure-mounted piston-tip-tilt piezo actuator that attaches to a common backplane structure that rests on jack screws atop the floating $16^{\prime} \times 4^{\prime}$ optical table. The segmented primary is the aperture stop and entrance pupil of SAINT. Two conic secondary mirrors are used to correct aberrations introduced by the off-axis parabolic collimator primary (labeled M1 in Fig. 2) and telescope primary. Construction of the segmented primary mirror has been completed, and was funded under GSFC Internal Research and Development (IRAD). In having done this, SAINT represents an effective leveraging strategy where costs for the TDEM effort are used mainly to integrate the SAINT components (the segmented telescope, FSM, and VNC), and perform the contrast measurements.
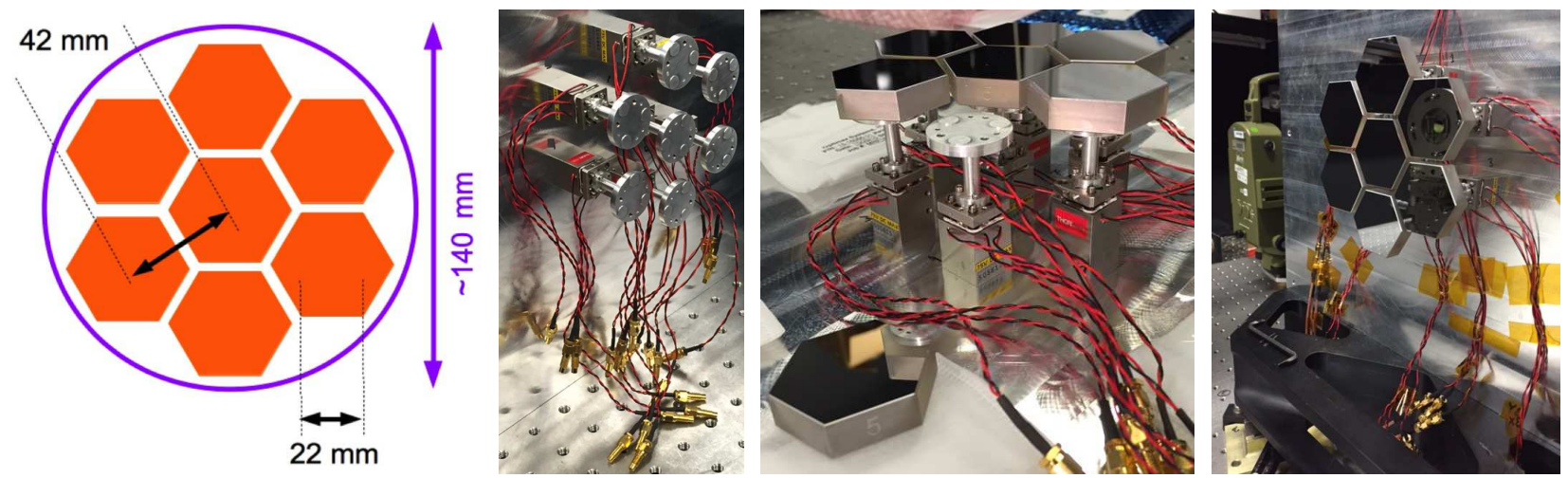

Figure 4. From left to right: The SAINT M1 aperture dimensions, fit-checking the mechanisms, mounts, and mirrors, and the mounted array following phasing and alignemet to the collimator and telescope.

\subsection{Visible Nulling Coronagraph}

Evaluations of segmented apertures, sensing/control, and telescope stability show that the VNC leads to a viable and manageable approach for a flight mission, ${ }^{14,15}$ including the discovery mission proposal for EPIC. ${ }^{5}$ To further evaluate the approach and sub-system-level technologies, a VNC laboratory testbed was developed at GSFC that has achieved milestones in nulling coronagraphy. Past work with the VNC has achieved repeatable contrasts of $5.5 \times 10-9$ at an IWA of $2 \lambda / D$ while operating under closed-loop control with a segmented deformable mirror. ${ }^{8,15}$ Unlike other traditional coronagraphs, this contrast result is the first high contrast demonstration with a segmented DM, and the deepest known nulling of any interferometer to date. The VNC testbed, as it stands now, has not been coupled to a telescope, but exists as a stand-alone laboratory testbed for evaluating nulling coronagraphy, sensing and control, and critical technologies including the DM and APS.

The VNC uses a combination of active and passive components to achieve the desired contrast at small IWA over a finite spectral bandpass. In its most simplistic form, it works by destructive and constructive interference: placing a star on the destructive interference central minimum (null) moves the stars light from the science channel to the bright pupil camera for wavefront and fine pointing control. Conversely, placing the planet on a constructive interference maximum allows transmission of the planet light to the dark science camera. The lower left panel of Fig. 2 shows a raytrace and schematic of modifications being made to VNC imaging and relay optics, including the addition of the FPS. Fore-optics re-image the pupil at the FSM to the coronagraph. The beamsplitter/combiner (BS/C) separate then recombine the two arms of the interferometer. A segmented deformable mirror (labeled PTT489 in the lower left panel of Fig. 2) corrects non-common path errors between the arms. The reference arm contains a delay mechanism which equalizes the path length between the two arms. Shutters and linear polarizers in each arm (not shown in the raytrace) are used to measure and balance amplitude, respectively. ${ }^{8,15}$ Aft-optics relay the bright and dark output beams to wavefront sensing and science cameras, respectively. 
Modifications to VNC fore-optics will be necessary for coupling to the segmented telescope. New additions to the fore-optics include the fine-steering mirror and tracking camera to form the fine pointing system for pointing offset correction and jitter compensation. The aft-optics will be simplified to remove the existing zoom lenses, folds, and periscopes to a straightforward relay from the dark channel output to the Lyot stop and then to the dark channel science camera. This will not only improve robustness and stability of the instrument, but also addresses chromatic aberration that was observed in the aft-optics used in previous demonstrations.

\section{MIRROR SEGMENT DESIGN AND ANALYSIS}

Several approaches were considered for fabricating the SAINT telescope mirror segments shown in Fig. 4. Each segment was polished from a $10 \mathrm{~mm}$ thick hexagonal piece of Zerodur with $\sim 22 \mathrm{~mm}$ edges, blocked in an array with $42 \mathrm{~mm}$ center-to-center spacing. The mirrors were designed to be bonded with RTV to an aluminum holder piece that has three $5 \mathrm{~mm}$ diameter raised bond pads. The sum of the masses of the mirror and adapter piece account for $40 \%$ of the piezo actuator load capacity. The optics are held horizontally with the optical axis perpendicular to gravity in their demonstration configuration.

Using measured segment surface data and known mechanical dimensions and material properties of mounts in a finite element analysis (FEA) enables full array static deformation and thermal expansion modeling for inclusion in a structural, thermal, optical (STOP) model of the system. A full STOP model of SAINT can be used to trace the model to the observatory scale where the telescope and the deformable mirror internal to the coronagraph are actively controlled to compensate through different dynamical environments. The model will be developed in in a piecewise manner for later integration. The tasks unfold as follows:

\subsection{Surface specifications and measured figure}

The SAINT primary mirror segments have a $4-\mathrm{m}$ concave radius of curvature and specified with an integrated wavefront error irregularity of $25 \mathrm{~nm}$ RMS within a hexagonal clear aperture that is $20 \mathrm{~mm}$ on edge. The specifications for segment surface irregularity is presented in Tab. 1.

\begin{tabular}{ccc}
\multicolumn{3}{c}{ Table 1. SAINT M1 optical surface specifications } \\
bin & wavelengths (mm) & spatial frequencies (cpa) \\
\hline 1 & $4.0-8.0$ & $5-10$ \\
2 & $8.0-10.0$ & $4-5$ \\
4 & $13.3-20.0$ & $2-3$ \\
5 & $>20.0^{*}$ & $<2^{*}$ \\
6 & total $^{*}$ & $<10^{*}$ \\
\hline${ }^{a}$ piston, tip, tilt, and power removed
\end{tabular}

An on-block measurement was taken to compare with individual segment measurements to quantify potential radius or curvature shifts associated with the deblocking process. Measurement of each of the individual segments was performed independently using a $\mathrm{f} / 38$ test piece. While it is preferable to observe the whole aperture in a single measurement, only irregularity over the individual segments is critical, as these errors propagate through the reference arm of the VNC uncorrected as the ultimate limit to the system end-to-end Strehl ratio. Radius variation between segments was measured to high precision, but not the actual value of the radius of curvature. One segment was set up as a reference surface, then replaced with another to measure the difference for irregularity with radius difference appearing as power measured in fringes. A 3-point fixture was used support the concave surfaces of the optics. The fixture was adjusted to align the first optic relative to an upward looking beam. Since each optic is spherical, centration is not critical. Measurement error arises if the fixture moves between measurements. After all segments were measured, the first piece was remeasured to be compared to its initial measurement and quantify how much the fixture moved during testing. The comparison indicated that error due to fixture instability was negligible and the measurement process was valid. Results of the measurements are presented in Fig. 5, with the right panel plotting measured surface errors for each segment in each of the spatial frequency bins listed in Tab. 1. Only segment number 4 (SN 4) exceeded the lowest spatial frequency bin specification. Measured higher spatial frequency bins were significantly lower than specification. It is expected 
that stress release following deblocking introduced the greatest amount of error for all segments, appearing as a cylindrical error.
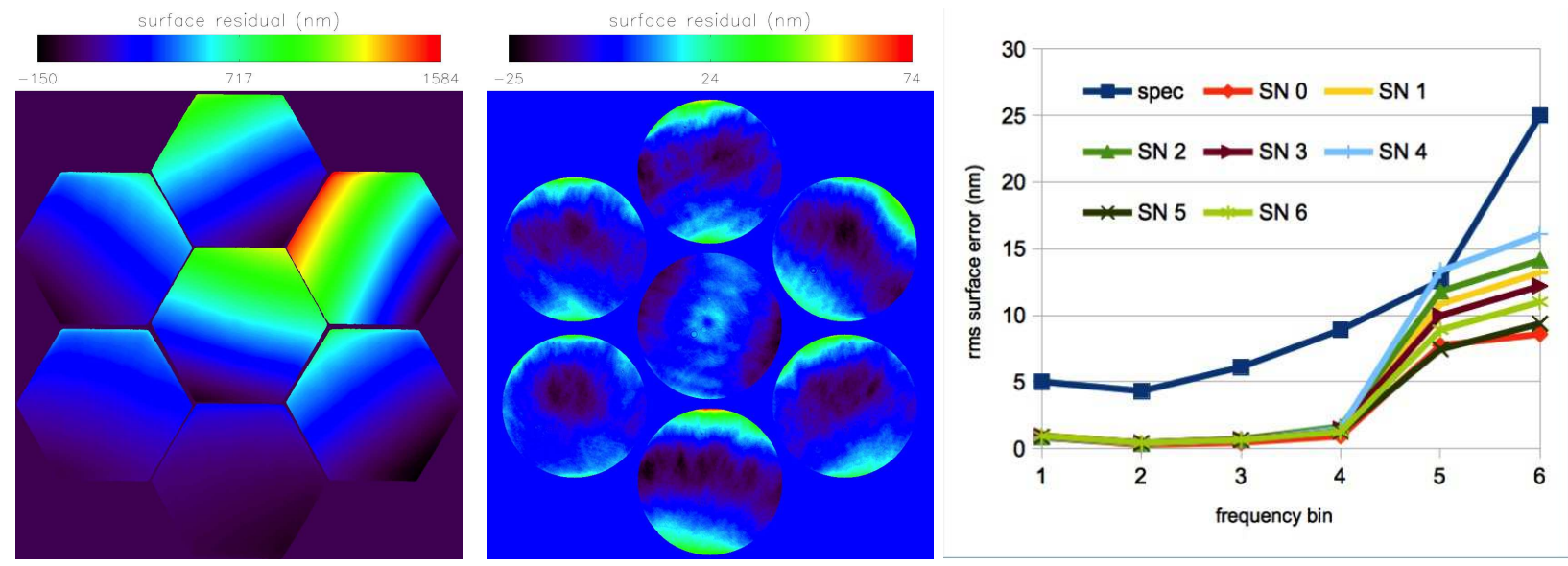

Figure 5. Left and center: independently measured segment surface data that is shown stiched together and with piston, tip, and tilt removed. Right: plots of measured surface error within specified spatial frequency bins (spec) for each segment identified by its segment number (SN) with 0 at the center and 1-6 clockwise starting at the top.

\subsection{Finite element analysis}

SAINT STOP modeling was initiated by quantifying mirror sag and deformation due to static gravity loading, bond layer stress, and thermal drift. The model components, meshes, and results are shown in Fig. 6. Surface polishing error and deformation maps may be used at a later date to perform detailed dynamic optical analysis of the SAINT telescope as the input to the VNC. Beyond the practical application of quantifying performance expectations in the lab, generating such a model would be a useful tool for better ensuring that future requirements for measuring system stability at picometer scales can be met, as well as helping to serve to interpret measurements. Verifying that the properties used in the physics-based models given in Tab. 2 accurately represent all properties of the system under test (dimensional, thermal, mechanical, electrical) is another step that must be taken in addition to quantifying the disturbances present in the operating environment (dynamic and steady-state electrical noise, gravitational field, as well as any gas, plasma, and radiation field dynamics). The inverse problem of model validation is not considered here, but in practice this must be done and is especially important when non-linear effects are a concern.
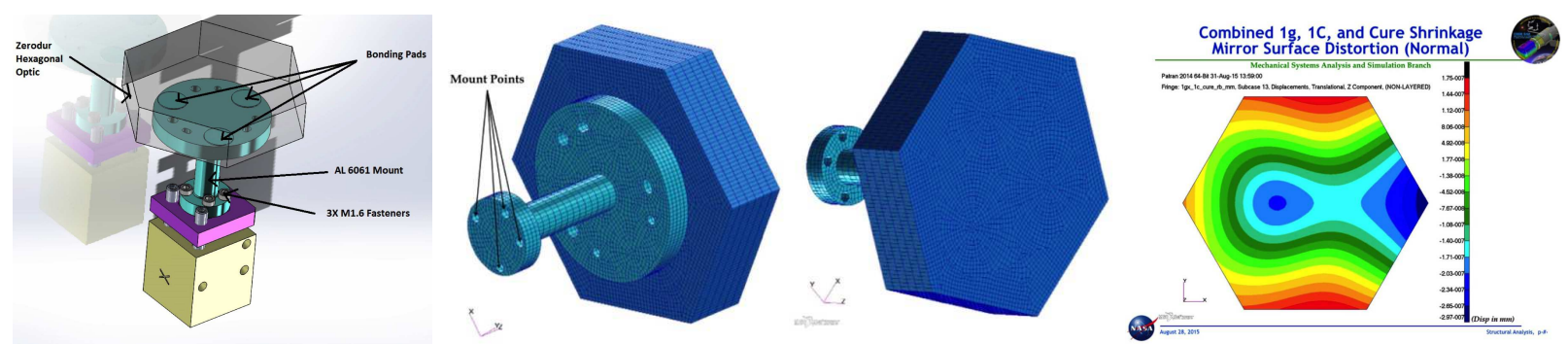

Figure 6. A finite element analysis model of the SAINT telescope primary segments including (left) the mirror segment, bonding and adapter piece, and actuator assembly, (center) the segment and adapter mesh and coordinate reference, and (right) the model results presented in Tab. 3

The full STOP analysis to be performed in future development should include (and would not necessarily be limited to) the following:

1. Perform an FEA of the assembled SAINT hexagonal packed primary mirror (PM). Goals: (i) Determine surface stress/strain due to force actuation, i.e., how much deformation does an actuator impart when 
Table 2. SAINT M1 model material properties and assumptions

\begin{tabular}{lcccccc} 
Material & $\mathrm{E}(\mathrm{psi})$ & $\nu$ & $\rho\left(\mathrm{lbs} / \mathrm{in}^{3}\right)$ & $\mathrm{CTE}\left(\mathrm{ppm} /{ }^{\circ} \mathrm{C}\right)$ & cure shrinkage $(\mathrm{in} / \mathrm{in})$ & component \\
\hline \hline Zerodur & $1.34 \times 10^{7}$ & 0.24 & 0.092 & 0.050 & - & mirror \\
Al 6061 T651 & $1.00 \times 10^{7}$ & 0.33 & 0.098 & 22.5 & - & post \\
RTV 566 & $6.09 \times 10^{2}$ & 0.45 & 0.055 & 2330 & 0.006 & bond layer \\
\hline
\end{tabular}

Table 3. SAINT M1 structural analysis results

\begin{tabular}{lcccccc} 
& \multicolumn{3}{c}{ Translation $(\mu \mathrm{m})$} & \multicolumn{3}{c}{ Rotation (arcsec) } \\
\hline Load case & $\mathrm{X}$ & $\mathrm{Y}$ & $\mathrm{Z}$ & $\mathrm{RX}$ & $\mathrm{RY}$ & $\mathrm{RZ}$ \\
\hline \hline 1g X & 1.523 & -0.001 & 0.000 & 0.01 & 10.07 & -0.01 \\
$1 \mathrm{~g} \mathrm{Y}$ & -0.001 & 1.539 & 0.003 & -10.14 & 0.01 & 0.00 \\
$1 \mathrm{~g} \mathrm{Z}$ & 0.000 & 0.004 & 0.036 & -0.02 & 0.00 & 0.00 \\
$1{ }^{\circ} \mathrm{C}$ bulk increase & -0.002 & -0.008 & 0.710 & -0.03 & -0.01 & 0.00 \\
Bond cure shrink & 0.000 & 0.000 & -2.009 & 0.00 & 0.00 & 0.00 \\
\hline
\end{tabular}

offloading gravity due to the mirror being mounted at $90^{\circ}$ relative to gravity (ii) Determine natural frequencies and structural eigenmodes of the mirrors mounted on their common backplane, which rests on the lab air table. (iii) Assess the effect of bulk temperature change and thermal gradients relative to local (x,y,z) directions.

2. Develop a mechanical model of the coronagraph under test including any aspheric or free-form mirror surfaces, also including incorporation of an existing segmented DM model. ${ }^{16}$ Goal: Determine the best mount points to offload gravity sag and what the deformations are as a function of thickness and material, including effects of isothermal and gradient temperature effects.

3. Develop a full mechanical model of the integrated SAINT system including all optical surfaces (first as rigid bodies, then with grids for deformations), and all mounts. Goal: Identify vibration modes of the coupled system and which optics and mounts are most sensitive in order to inform the design of closed-loop sensing and control mechanisms and establish a system-level error budget.

Ultimately this work would be used to build a full STOP model that would feed into a brassboard groundor sub-orbital instrument, and most importantly, to show a path forward for the VNC paired with a future large space telescope.

\section{ARRAY ALIGNMENT AND STABILITY MEASUREMENTS}

Following bonding and assembly of the individual segments and actuators on their common backplane, the SAINT segmented primary was coarse aligned on an optical bench large enough to accommodate using an illumination source $4 \mathrm{~m}$ away from the array at its center of curvature. This initial alignment was performed by only powering the piezo actuators of one segment at a time to aid in setting coarse piston. After coarse alignment, the array was moved to its demonstration location on the SAINT optical table to perform an intermediate alignment step with all piezos powered to their mid-stroke settings. Once the array was phased, it was aligned to the SAINT collimator, followed by alignment of the telescope primary, the positions of which were located using a combination of coordinate measuring machine and theodolite-assisted adjustments. Once the initial alignment of the foreoptics was completed, initial stability data were collected. Further details of the array alignment and stability measurements are described below in Sec. 5.1 and Sec. 5.2, respectively.

\subsection{Array alignment}

Divergent laser, diode, and supercontinuum fiber source beams were used alternately to fill the segment array for either easy viewing or coherent alignment of piston, tip, and tilt for each segment. The light reflected from the array was picked off by a beamsplitter near the source that redirected the returned light to a projection screen used to observe the spots associated with each actuator as it was adjusted. Coarse adjustment in tip and 
tilt made use of 100 TPI actuator screws built into the 3-degrees of freedom (DOF) stages to concentrate the spots into an area that fit on a large format detector. A simulation of what is observed at this stage is shown in Fig. 7. The segments were then phased one at a time, making small adjustments to each segment's piston screw, realigning tip and tilt, then scanning through the piezo piston range to locate the white light fringe packet relative to the central segment. Coarse adjustment using the 100 TPI screws gives 1 um piston and 15 arcsec tip/tilt resolution prior to handing-off to piezo fine controls that have $7 \mu \mathrm{m}$ piston and $120 \mathrm{arcsec}$ tip/tilt range.
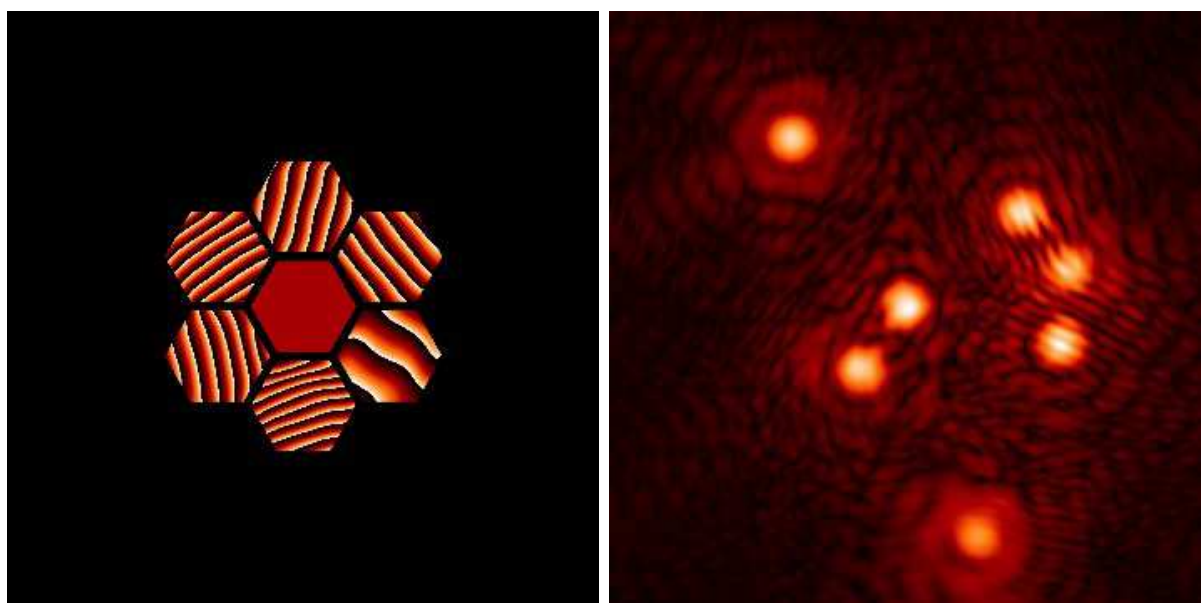

Figure 7. A simulation of surface errors and residual tilt fringes observed in the pupil plane to be removed through piezo actuation (left), and the correspnonding PSFs associated with each segment prior to coherent phasing (right).

Once coarse adjustment of the segment actuators was completed, piezo actuation was used to bring each of the six outer segments into fine piston, tip, and tilt alignment by eye. This intermediate step was performed using a mask consisting of two circular subaptures as shown in Fig. 8. The segment pair mask consisted of $32 \mathrm{~mm}$ diameter circlular subapertures spaced at $42 \mathrm{~mm}$. The pairwise approach minimizes confusion for this human-in-the-loop step that will become an automated routine. This process was iterated with adjustments to the coarse actuation screws to achieve array fine alignment such that the voltage matrix range of all 21DOF (tip, tilt, and piston for seven segments) was within $37.5 \pm 7.5 \mathrm{~V}$, corresponding to the amplifier midrange voltage \pm one-tenth of the control range.
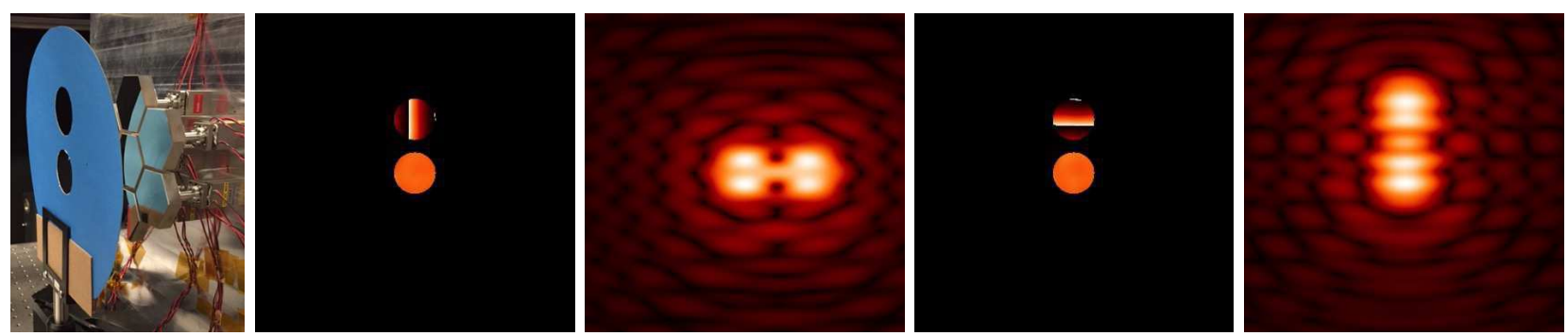

Figure 8. A mask consisting of a pair of circular subapertures (left) is used to align the outer segments to the central segmented, one at a time using piezo actuation. Simulated pupils and focal plane images show approximately a full wave of tip and tilt.

A set of simulated pupils and PSFs is shown in Fig. 9 for the paired subapertures, and in Fig. 10 the full array of seven segments, each with only one segment perturbed in either pure tip or tilt. The offsets shown in the simulations represent $0, \lambda / 2$, and $\lambda \mathrm{PV}$ tip/tilt surface errors $(0, \lambda, 2 \lambda$ tip/tilt wavefront error). Corresponding measured data is also shown for each case using a source with a $40 \mathrm{~nm}$ bandpass filtered centered at $650 \mathrm{~nm}$. Measured data corresponds to $0.0,1.5$, and $3.0 \mathrm{~V}$ amplifier control, which give $0,2.4$ and 4.8 arcsec rotations according to device specifications. The diffraction radius of each segment's PSF is $\sim \lambda / D=4.2$ arcsec, and half the extent of the phased PSF in the narrow direction is $\lambda / 2 B=1.6$ arcsec. The discrepancies between simulated and measured PSFs are due to static errors in array alignment, lab turbulence, and mechanical disturbances that will be corrected and mitigated through the development and incorporation of closed-loop sensing and control that will automatically generate a response matrix for all 21DOF. Rather than drive to the location of any single 
segment (e.g., the central segment) a final phased solution will target an ensemble average control voltage setting equal to the amplifiers' midrange setting of $37.5 \mathrm{~V}$.
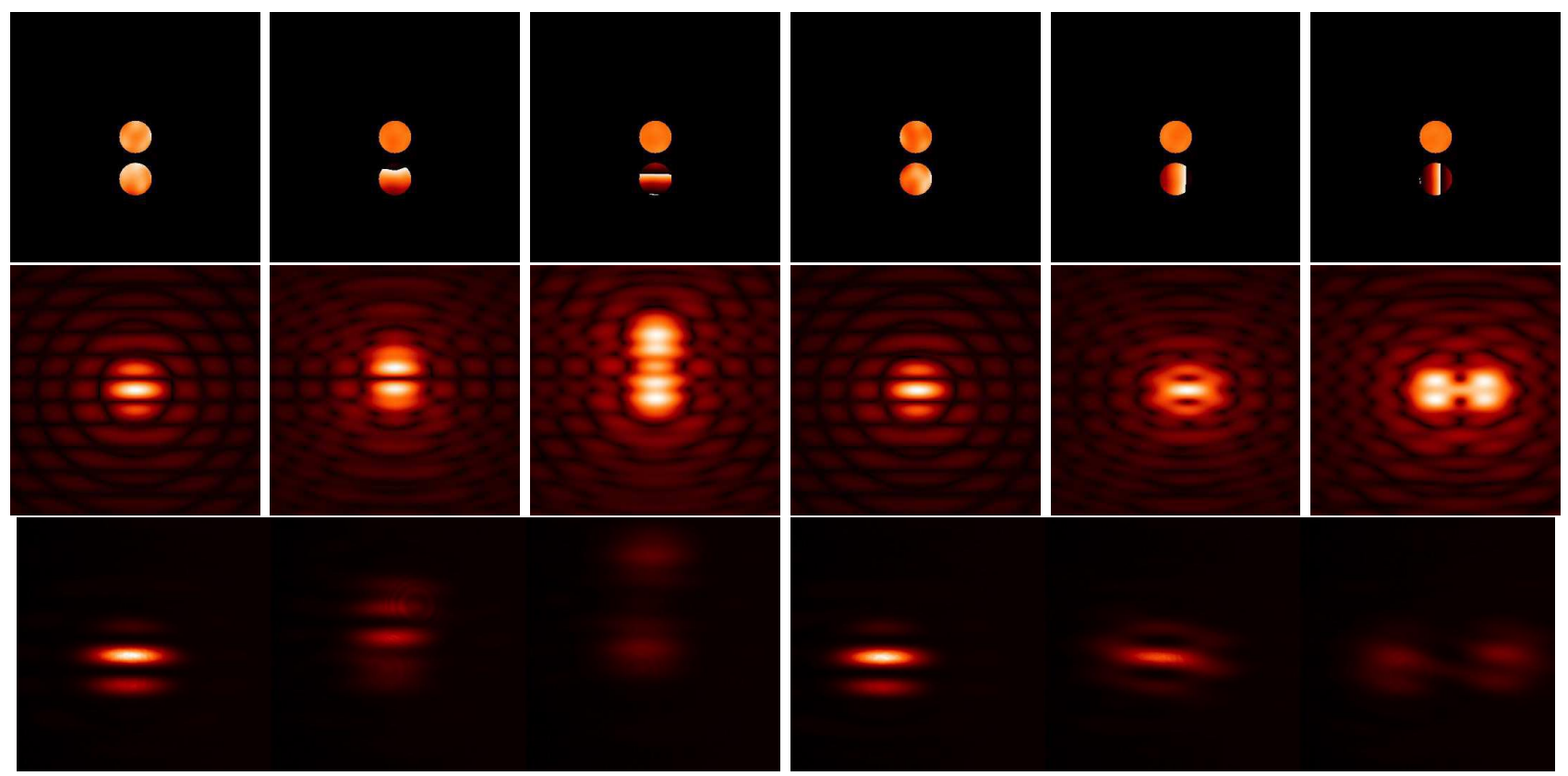

Figure 9. Simulated circular subaperture pair pupils (top), PSFs (center), and the first corresponding measured PSFs (below) generated using the SAINT collimator and telescope. The discrepancies between simulated and measured PSFs are attributed to static errors in array alignment and lab environmental disturbances that are to be actively corrected.
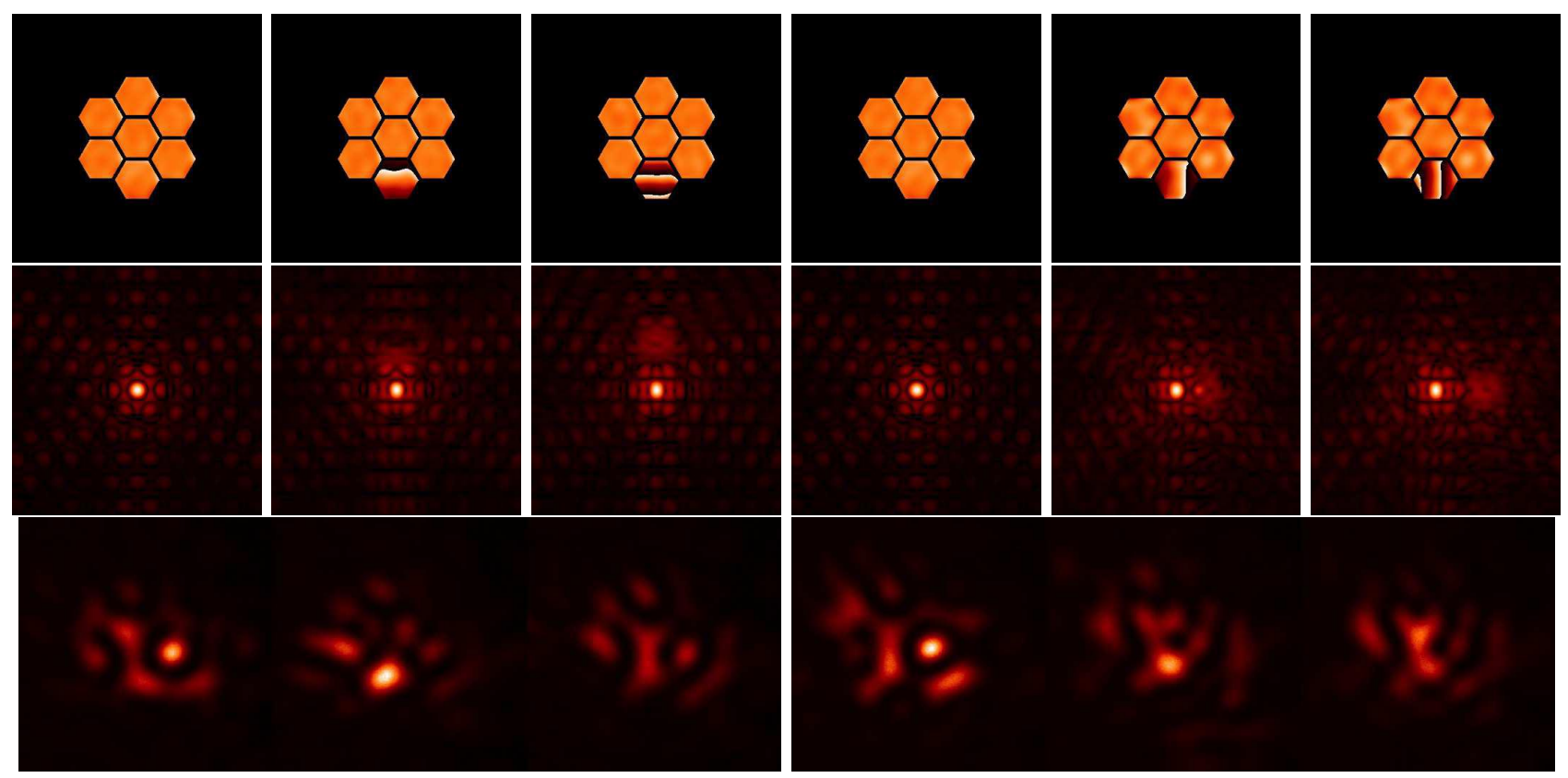

Figure 10. The same as Fig. 9 for the full SAINT telescope primary.

Edge sensors ${ }^{17-19}$ could be used to automate fast sensing and phasing of the segmented array by sampling two segments simultaneously with the same optic to form a coherent image or dispersed fringe. A challenge to using this approach as shown for the SAINT telescope primary is that a large fraction of the mirror surfaces that each lens would sample would be outside the segments' clear apertures (see Fig. 11). Using three larger lenses to 
sample three sets of three mirrors with the central segment being sampled by each lens could also be explored. In either case, tip and tilt would be removed across the array before performing a piston correction.
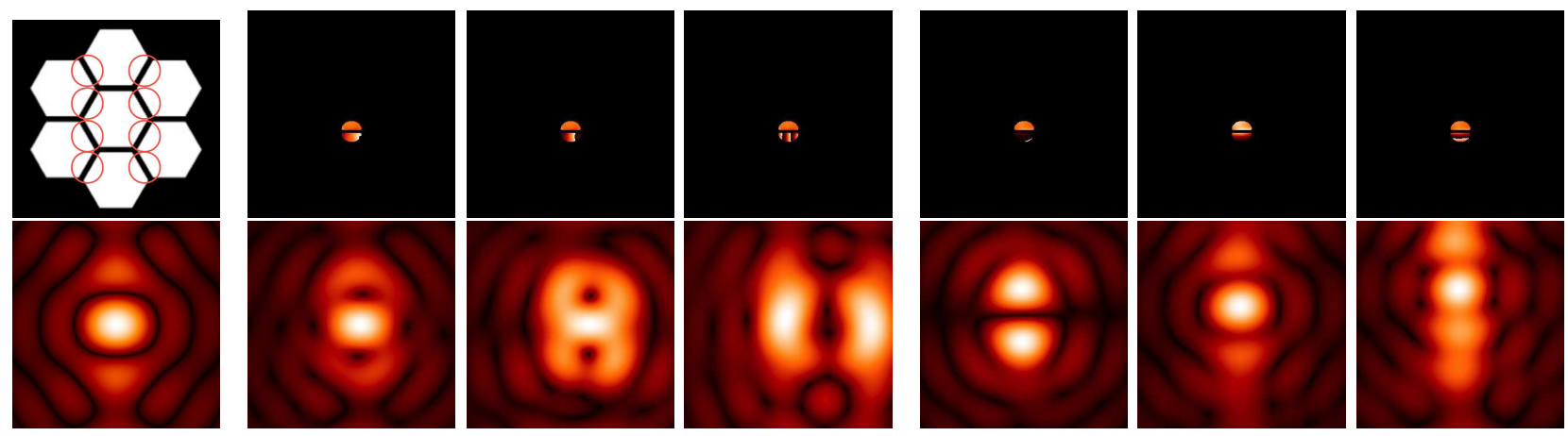

Figure 11. A modified Shack-Hartman approach to sensing piston, tip, and tilt errors across the SAINT M1 array using lenses that span segment gaps, shown in the upper left as red circles. The simulated lenslet pupils and PSFs correspond to $\lambda / 2, \lambda / 2$, and $2 \lambda$ WFE. The PSFs are autoscaled.

\subsection{Stability measurements}

Following the initial alignment of the segmented primary mirror array along with its secondary and the source collimator optics, baseline stability measurements were performed to characterize long period alignment drifts as well as high frequency turbulence and jitter. The first and last frames taken in both the low and high frequency regimes are shown in Fig. 12. A more detailed analysis that what is found here will be performed at a later date once all plate scales are fixed and mounts and optics have been locked.
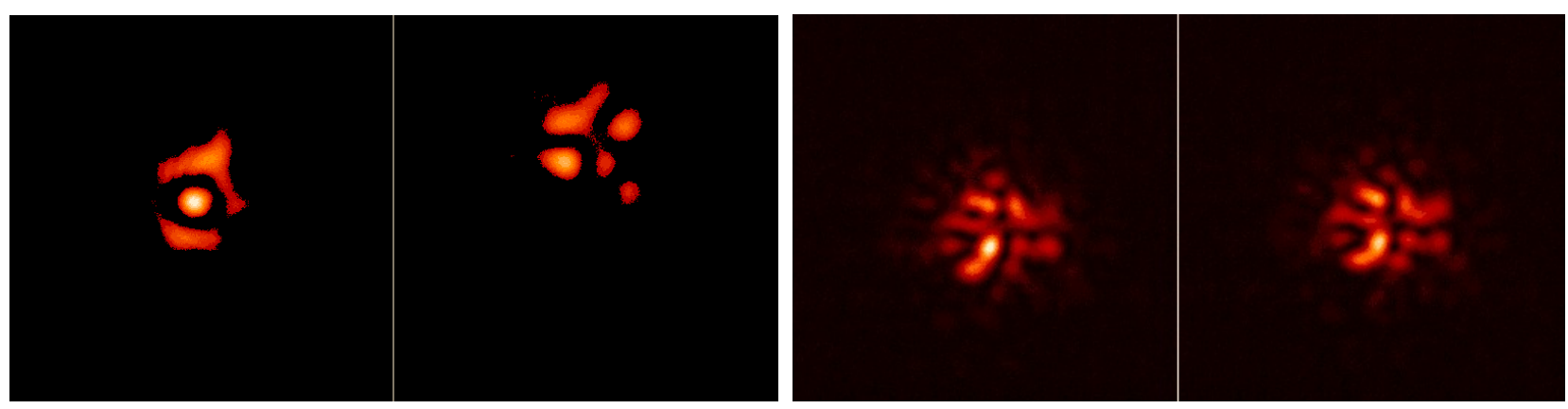

Figure 12. The first and last frames captured in the (left) low frequency drift and (right) high frequency jitter measurements.

For these first measurements following initial alignment, long period drift was observed over a twenty-four hour period at a sampling rate of one frame every five minutes. The left top and bottom figures of Fig. 13 show the centroid drifting by a magnitude of approximately 40 pixels. Large excursions due to impulse-type perturbations may be reduced by averaging several frames. High frequency jitter was measured by sampling at $800 \mathrm{fps}$ over a period of two minutes for a total of 94000 frames. The plate scale of these data are $\approx 4$ pixels per $\lambda / D$, where $D \approx 120 \mathrm{~mm}$ for the full array. The power spectrum of the high frequency data shows an as of yet unidentified peak near $28 \mathrm{~Hz}$ in addition to a wall current peak at $60 \mathrm{~Hz}$. The next step before performing further measurements will be record data prior to and following the addition of an enclosure to reduce jitter attributed to lab turbulence. The enclosure will use panels that minimize transmission of acoustic noise.

Slow drift in segment to segment alignment is evidenced by speckle intensity variation visible in the first and final frames of both the low and high frequency data shown in Fig. 12). This proxy for intersegment misalignments may be used to identify and mitigate sources of drift, e.g., piezo amplifier control voltages that can be monitored independently. The plots in Fig. 14 show the normalized time evolution and PSD of peak pixel intensity and the standard deviation of all pixel intensities. These data and another set of measurements to be recorded prior 

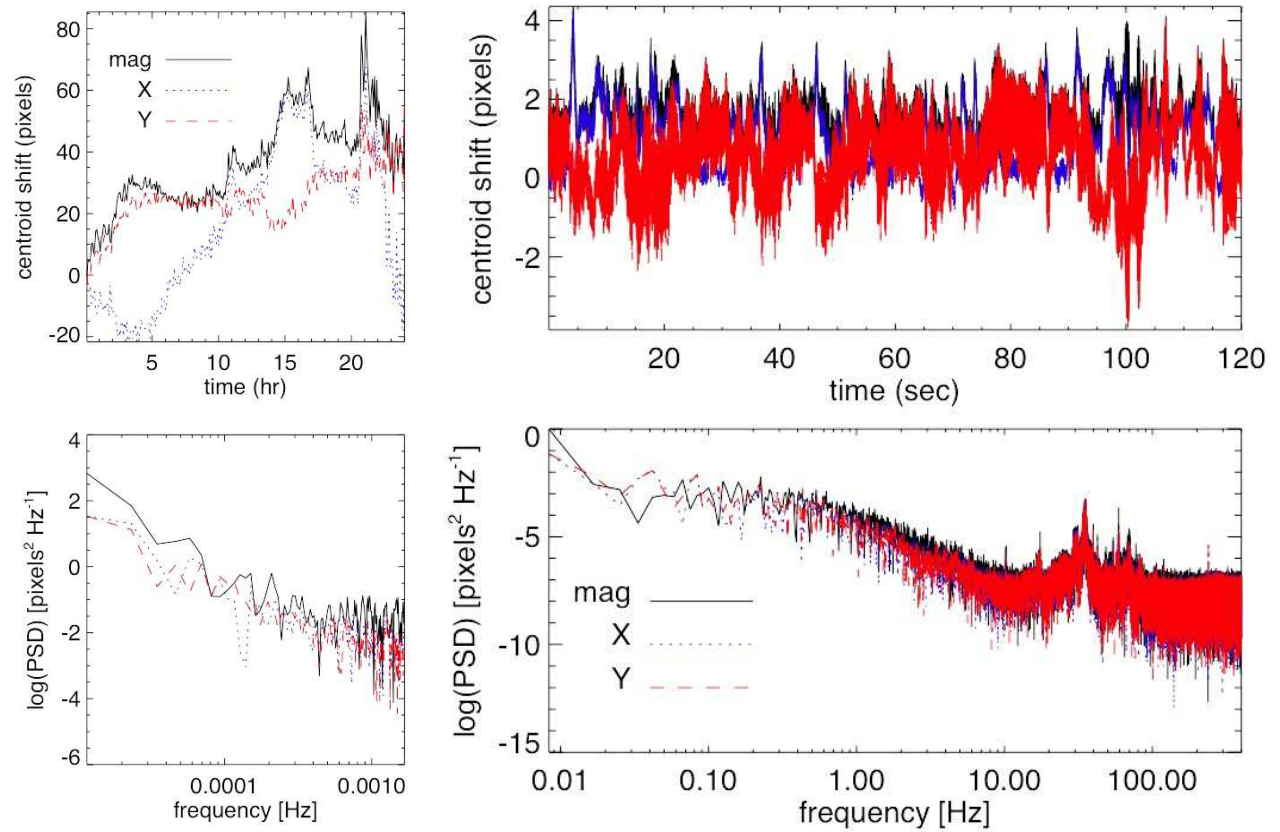

Figure 13. PSF centroid drift and jitter. Left top and bottom: long period centroid drift and the corresponding power spectrum; Right top and bottom: high frequency centroid shift and the corresponding power spectrum.

to enclosing the source, collimator, and telescope optics will be used to establish an open-loop baseline prior to closing the loop using, e.g. a Shack-Hartmann edge sensor as described in Sec. 5.1.
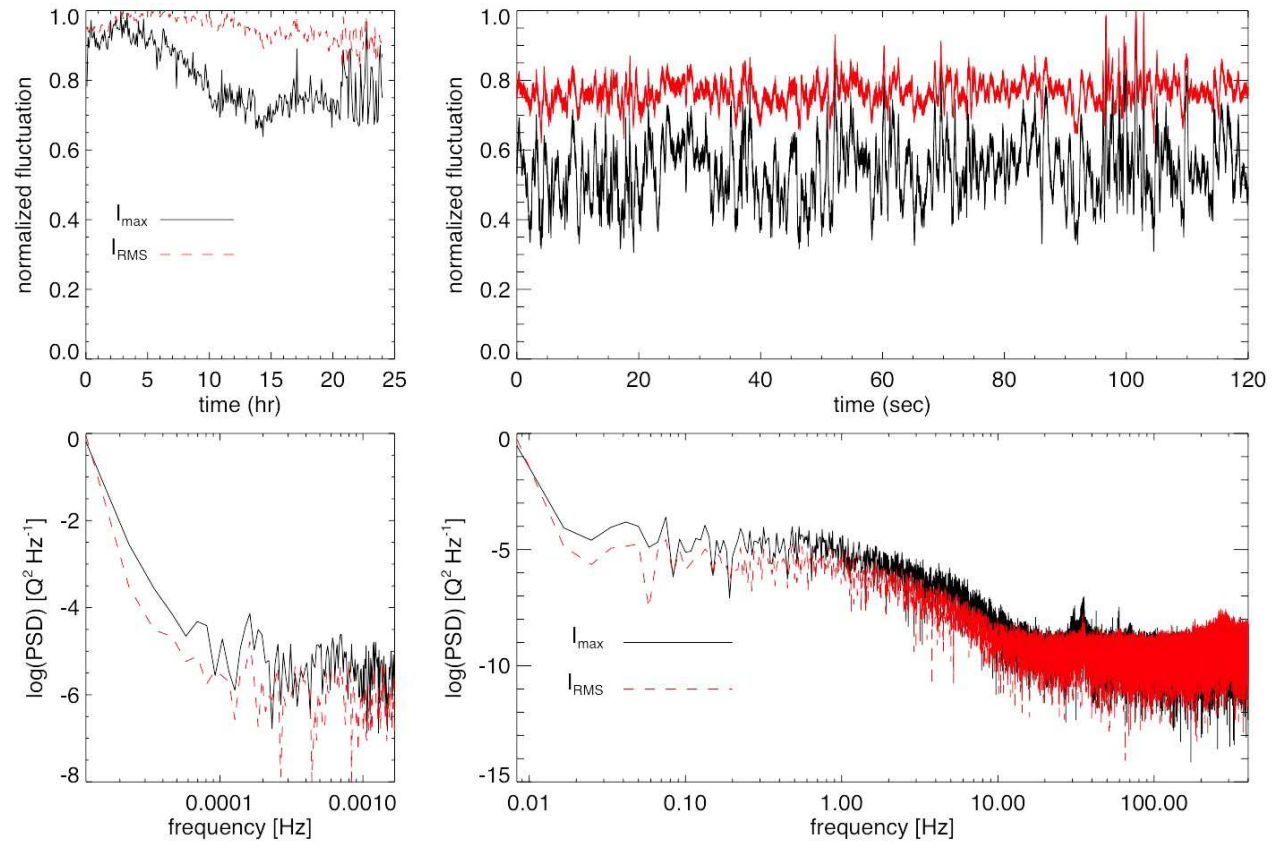

Figure 14. PSF stability characterized by normalized peak pixel intensity and RMS pixel intensity. Left top and bottom: long period fluctuation and the corresponding power spectrum; Right top and bottom: long period fluctuation and the corresponding power spectrum. 


\section{SUMMARY}

SAINT aims to advance coronagraphic techniques for future segmented aperture telescopes. SAINT integrates the VNC with an actively controlled hexagonal array segmented mirror telescope via a fine pointing system. The first SAINT effort will culminate in a demonstration of high-contrast imaging with a complex aperture enabled by sequential wavefront control. This SAT/TDEM-funded work specifically targets achieving a contrast of $10^{-8}$ over a $1 \lambda / D$ diameter circular area centered at $4 \lambda / D$ using a source spectral bandpass of $20 \mathrm{~nm}$ centered on $633 \mathrm{~nm}$. A stretch goal will be to achieve $10^{-9}$ contrast at a smaller IWA, possibly $\sim 3 \lambda / D$, and a broader bandpass of $40 \mathrm{~nm}$. Achieving this Milestone will further advance coronagraphy towards being adaptable to arbitrary apertures, thereby freeing up the necessity of requiring unobscured off-axis telescopes for direct imaging of exoplanets. SAINT will be taking a significant step towards coronagraphy becoming viable for any telescope.

\section{ACKNOWLEDGMENTS}

The authors acknowledge support from NASA/Goddard Space Flight Center Internal Research and Development (IRAD), and from the NASA Strategic Astrophysics Technology (SAT) Technology Development for Exoplanet Missions (TDEM) program.

\section{In memory of Richard G. Lyon (1958-2016)}

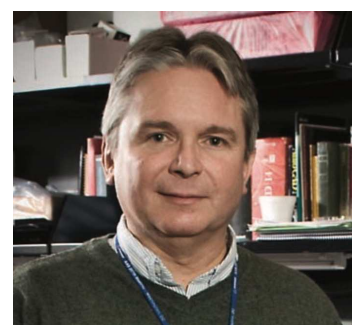

\section{REFERENCES}

[1] Stark, C. C., Roberge, A., Mandell, A., Clampin, M., Domagal-Goldman, S. D., McElwain, M. W., and Stapelfeldt, K. R., "Lower Limits on Aperture Size for an ExoEarth Detecting Coronagraphic Mission," Astrophys. J. 808, 149 (Aug. 2015).

[2] N'Diaye, M., Soummer, R., Pueyo, L., Carlotti, A., Stark, C. C., and Perrin, M. D., "Apodized Pupil Lyot Coronagraphs for Arbitrary Apertures. V. Hybrid Shaped Pupil Designs for Imaging Earth-like planets with Future Space Observatories," Astrophys. J. 818, 163 (Feb. 2016).

[3] Guyon, O., Hinz, P. M., Cady, E., Belikov, R., and Martinache, F., "High Performance Lyot and PIAA Coronagraphy for Arbitrarily Shaped Telescope Apertures," Astrophys. J. 780, 171 (Jan. 2014).

[4] Hicks, B. A., "Exoplanet detection and characterization via parallel broadband nulling coronagraphy," J. Ast. Tel. Inst. \& Sys. 2, 011015 (Jan. 2016).

[5] Clampin, M., Melnick, G., Lyon, R., Kenyon, S., Sasselov, D., Tolls, V., Ford, H., Golimowski, D., Petro, L., Hartig, G., Sparks, W., Illingworth, G., Lin, D., Seager, S., Weinberger, A., Harwit, M., Marley, M., Schneider, J., Shao, M., Levine, M., Ge, J., and Woodruff, R., "Extrasolar planetary imaging coronagraph (EPIC)," Proc. SPIE 6265 (July 2006).

[6] Postman, M., Argabright, V., Arnold, B., Aronstein, D., Atcheson, P., Blouke, M., Brown, T., Calzetti, D., Cash, W., Clampin, M., Content, D., Dailey, D., Danner, R., Doxsey, R., Ebbets, D., Eisenhardt, P., Feinberg, L., Fruchter, A., Giavalisco, M., Glassman, T., Gong, Q., Green, J., Grunsfeld, J., Gull, T., Hickey, G., Hopkins, R., Hraba, J., Hyde, T., Jordan, I., Kasdin, J., Kendrick, S., Kilston, S., Koekemoer, A., Korechoff, B., Krist, J., Mather, J., Lillie, C., Lo, A., Lyon, R., McCullough, P., Mosier, G., Mountain, M., Oegerle, B., Pasquale, B., Purves, L., Penera, C., Polidan, R., Redding, D., Sahu, K., Saif, B., Sembach, K., Shull, M., Smith, S., Sonneborn, G., Spergel, D., Stahl, P., Stapelfeldt, K., Thronson, H., Thronton, G., Townsend, J., Traub, W., Unwin, S., Valenti, J., Vanderbei, R., Werner, M., Wesenberg, R., Wiseman, 
J., and Woodgate, B., "Advanced Technology Large-Aperture Space Telescope (ATLAST): A Technology Roadmap for the Next Decade," ArXiv e-prints (Apr. 2009).

[7] Shao, M., Bairstow, S., Martin Levine, B., Vasisht, G., Lane, B. F., Vasudevan, G., Woodruff, R., Samuele, R., Wynn, J., Clampin, M., Lyon, R., and Guyon, O., "DAVINCI, a diluter aperture visible nulling coronagraphic instrument," Proc. SPIE 7013 (July 2008).

[8] Clampin, M., Lyon, R., Petrone III, P., Mallik, U., Bolcar, M., Madison, T., and Helmbrecht, M., "Visible nulling coronagraph technology maturation High contrast imaging and characterization of exoplanets," tech. rep., NASA/Technology Development for Exoplanet Missions Final Report, JPL Document D-80950, https://exep.jpl.nasa.gov/technology/Clampin_Report_FINAL.pdf (2013).

[9] Hicks, B., Bolcar, M., Lyon, R., Clampin, M., Madison, T., Mallik, U., Petrone III, P., and Helmbrecht, M., "Technology milestone \#2 final report: Achromatic visible nulling coronagraph technology maturation," tech. rep., NASA/Technology Development for Exoplanet Missions, JPL Document D-1547413, https://exoplanets.nasa.gov/exep/technology/TDEM-awards/ (2016).

[10] Bolcar, M. R., Balasubramanian, K., Crooke, J., Feinberg, L., Quijada, M., Rauscher, B., Redding, D., Rioux, N., Shaklan, S., Stahl, H. P., Stahle, C., and Thronson, H., "Technology gap assessment for a future large-aperture ultraviolet-optical-infrared space telescope," J. Ast. Tel. Inst. ES Sys. 2, 041209 (2016).

[11] Morgan, R. and Siegler, N., "Initial look at the coronagraph technology gaps for direct imaging of exoearths," Proc. SPIE 9605, 96052I (Sept. 2015).

[12] Carpenter, K. G., Lyon, R. G., Schrijver, C., Karovska, M., and Mozurkewich, D., "Direct UV/optical imaging of stellar surfaces: the Stellar Imager Vision Mission," Proc. SPIE 6687, 66870G (Sept. 2007).

[13] Lyon, R. G., Carpenter, K. G., Liu, A., Petrone, P., Dogoda, P., Reed, D., and Mozurkewich, D., "Wavefront sensing and closed-loop control for the Fizeau interferometry testbed," Proc. SPIE 6687, 0 (Sept. 2007).

[14] Lyon, R. G., Clampin, M., Woodruff, R., Vasudevan, G., Shao, M., Levine, M., Melnick, G., Tolls, V., Petrone, P., Dogoda, P., Duval, J., and Ge, J., "Visible Nulling Coronagraphy for Exo-Planetary Detection and Characterization," in [IAU Colloq. 200: Direct Imaging of Exoplanets: Science and Techniques], Aime, C. and Vakili, F., eds., 345-352 (2006).

[15] Lyon, R. G., Clampin, M., Petrone, P., Mallik, U., Madison, T., and Bolcar, M. R., "High contrast vacuum nuller testbed (VNT) contrast, performance, and null control," Proc. SPIE 8442 (Sept. 2012).

[16] Aguayo, E. J., Lyon, R., Helmbrecht, M., and Khomusi, S., "FEM correlation and shock analysis of a VNC MEMS mirror segment," Proc. SPIE 9143, 91435C (Aug. 2014).

[17] Shi, F., Chanan, G., Ohara, C., Troy, M., and Redding, D. C., "Experimental Verification of Dispersed Fringe Sensing as a Segment Phasing Technique using the Keck Telescope," Appl. Opt. 43, 4474-4481 (Aug. 2004).

[18] Kanneganti, S., McLeod, B. A., Ordway, M. P., Roll, J. B., Shectman, S. A., Bouchez, A. H., Codona, J., Eng, R., Gauron, T. M., Handte, F., Norton, T. J., Streechon, P., and Weaver, D., "A prototype phasing camera for the Giant Magellan Telescope," Proc. SPIE 8447, 844752 (July 2012).

[19] Surdej, I., Romnée, E., Bastaits, R., Preumont, A., Yaitskova, N., and Noethe, L., "Influence of atmospheric turbulence on the Zernike phase contrast method and the first steps towards the phasing of segmented deformable mirrors," Proc. SPIE 8447, 84476L (July 2012). 